\title{
ON THE LOW REGULARITY SOLUTIONS FOR A MODIFIED TWO-COMPONENT CAMASSA-HOLM SHALLOW WATER SYSTEM
}

\author{
XINGXING LIU and ZHAOYANG YIN \\ Department of Mathematics, Sun Yat-sen University, 510275 Guangzhou, China \\ e-mail: liuxingxing123456@163.com,mcsyzy@mail.sysu.edu.cn
}

(Received 23 June 2010; accepted 30 November 2010; first published online 10 March 2011)

\begin{abstract}
We first study the regularised version of a modified two-component Camassa-Holm shallow water system and obtain the energy estimates of the corresponding approximate solutions. Then, we present a sufficient condition which guarantees that these approximate solutions converge to a low regularity weak solution of the modified two-component Camassa-Holm shallow water system.
\end{abstract}

2010 Mathematics Subject Classification. 35G25, 35L05.

1. Introduction. In this paper, we consider the following modified twocomponent Camassa-Holm shallow water system [23]:

$$
\begin{cases}m_{t}+u m_{x}+2 m u_{x}=-\sigma \rho \bar{\rho}_{x}, & t>0, x \in \mathbb{R}, \\ \rho_{t}+(\rho u)_{x}=0, & t>0, x \in \mathbb{R}, \\ m(0, x)=m_{0}(x), & x \in \mathbb{R}, \\ \rho(0, x)=\rho_{0}(x), & x \in \mathbb{R},\end{cases}
$$

where $m=u-u_{x x}$ and $\rho=\left(1-\partial_{x}^{2}\right)\left(\bar{\rho}-\bar{\rho}_{0}\right)$.

The Camassa-Holm equation is a well-known integrable equation describing the unidirectional propagation of shallow water waves over a flat bottom $[\mathbf{2}, \mathbf{7}, \mathbf{1 5}, \mathbf{2 4}]$. It is also a model for the propagation axially symmetric waves in hyperelastic rods [13]. It has a bi-Hamiltonian structure [5] and is completely integrable [2, 6]. Its solitary waves are peaked [3], capturing thus the shape of solitary wave solutions to the governing equations for water waves [10]. The orbital stability of the peaked solutions is proved in [12]. In fact, a lot of works have been carried out to study the Cauchy problem and initial-boundary value problem for the Camassa-Holm equation $[\mathbf{8}, \mathbf{9}, \mathbf{1 4}, \mathbf{1 7}, \mathbf{1 8}, \mathbf{2 6}$, 28].

The two-component Camassa-Holm equation was recently introduced by Chen et al. in [4], Flaqui in [19] and Constantin and Ivanov in [11]. The variable $u(x, t)$ describes the horizontal velocity of the fluid and the variable $\rho(x, t)$ is in connection with the horizontal deviation of the surface from equilibrium, all measured in dimensionless units [11]. The mathematical properties of two-component CamassaHolm equation have been studied in many works cf. $[4,11,16,20]$.

The Camassa-Holm equation was recently extended to a two-component integrable system by combing its integrability property with compressibility, or free-surface elevation dynamics in its shallow-water interpretation [11, 23]. The system (1.1) introduced recently by Holm et al. in [23] is a modified version of the 
two-component integrable system to allow a dependence on the average density $\bar{\rho}$ as well as the pointwise density $\rho$, cf. [23]. The modified two-component Camassa-Holm system is written in terms of velocity $u$ and locally averaged density $\bar{\rho}$ and $\bar{\rho}_{0}$ is taken to be constant. Recently, Holm and Ivanov [22] gave a new interpretation of this system. The modified $\mathrm{CH} 2$ system in $1 D$ has been shown to be equivalent to the original $E P D i f f\left(H^{1}\right)$ equation in $2 D$ coordinates $\left(x_{1}, x_{2}\right) \in R^{2}$.

Recently, the modified two-component Camassa-Holm system was studied in [21]. Their work established the local well-posedness and derived precise blow-up scenarios, and proved that the system has strong solutions which blow up in finite time.

Motivated by [21] and [26], the purpose of this paper is to study the low regularity solutions for the modified two-component Camassa-Holm system (1.1). For the sake of convenience, we always assume $\sigma=1$ in the remainder of the paper.

Our paper is organised as follows. In Section 2, we summarise our basic notations and recall some required equalities. In Section 3, we present some results of the regularised modified two-component Camassa-Holm system. In Section 4, we study the low regularity solutions for a modified two-component Camassa-Holm system.

2. Preliminaries. We begin by summarising our basic notations. For any real number $s$, we let $H^{s}=H^{s}(\mathbb{R})$ denote the Sobolev space consisting of all tempered distributions $f$ such that

$$
\|f\|_{H^{s}}=\left(\left.\int_{-\infty}^{\infty}\left(1+|\xi|^{2}\right)^{s} \widehat{\mid f}(\xi)\right|^{2} d \xi\right)^{1 / 2}<\infty .
$$

For any function $u=u(x, t): \mathbb{R} \times[0, T) \rightarrow \mathbb{R}$ of two variables with $T>0$, we denote its Fourier transform, $L^{p}$-norm and $H^{s}$-norm with respect to $x$ by $\widehat{u}=\widehat{u}(\xi, t),\|u\|_{L^{p}}=$ $\|u(\cdot, t)\|_{L^{p}}$ and $\|u\|_{H^{s}}=\|u(\cdot, t)\|_{H^{s}}$, respectively. The integral operator $\Lambda=\left(I-\partial_{x}^{2}\right)^{1 / 2}$ will play a key role. We let $[A, B]$ denote the commutator of linear operator $A$ and $B$.

Next, we will give a few basic inequalities.

Lemma 2.1. [25]. If $r>0$, then $H^{r} \cap L^{\infty}$ is an algebra. Moreover,

$$
\|f g\|_{H^{r}} \leq c\left(\|f\|_{L^{\infty}}\|g\|_{H^{r}}+\|f\|_{H^{r}}\|g\|_{L^{\infty}}\right),
$$

where $c$ is a constant depending only on $r$.

LEMMA 2.2. [25]. If $r>0$, then

$$
\left\|\left[\Lambda^{r}, f\right] g\right\|_{L^{2}} \leq c\left(\left\|\partial_{x} f\right\|_{L^{\infty}}\left\|\Lambda^{r-1} g\right\|_{L^{2}}+\left\|\Lambda^{r} f\right\|_{L^{2}}\|g\|_{L^{\infty}}\right),
$$

where $c$ is a constant depending only on $r$.

Then, we shall reformulate the problem (1.1). With $m=u-u_{x x}, \rho=\gamma-\gamma_{x x}$ and $\gamma=\bar{\rho}-\overline{\rho_{0}}$, we can rewrite the system (1.1) as follows:

$$
\begin{cases}u_{t}-u_{t x x}+3 u u_{x}=F, & t>0, x \in \mathbb{R}, \\ \gamma_{t}-\gamma_{x x t}+\left(\left(\gamma-\gamma_{x x}\right) u\right)_{x}=0, & t>0, x \in \mathbb{R}, \\ u(0, x)=u_{0}(x), & x \in \mathbb{R}, \\ \gamma(0, x)=\gamma_{0}(x), & x \in \mathbb{R},\end{cases}
$$

where $F=2 u_{x} u_{x x}+u u_{x x x}-\gamma_{x}\left(\gamma-\gamma_{x x}\right)$. 
3. A priori estimates. In this section, we will consider the initial-value problem for a regularised version of the system (2.3):

$$
\begin{cases}u_{t}-u_{t x x}+\varepsilon u_{x x x x t}+3 u u_{x}=F, & t>0, x \in \mathbb{R} \\ \gamma_{t}-\gamma_{x x t}+\varepsilon \gamma_{x x x x t}+\left(\left(\gamma-\gamma_{x x}\right) u\right)_{x}=0, & t>0, x \in \mathbb{R}, \\ u(0, x)=u_{0}(x), & x \in \mathbb{R}, \\ \gamma(0, x)=\gamma_{0}(x), & x \in \mathbb{R},\end{cases}
$$

where $F=2 u_{x} u_{x x}+u u_{x x x}-\gamma_{x}\left(\gamma-\gamma_{x x}\right)$ and $\varepsilon$ is a constant with $0<\varepsilon<1 / 4$.

Lemma 3.1. For any $0<\varepsilon<1 / 4$ and any $s \geq 1$, the integral operator

$$
\mathcal{D}=\left(\mathrm{I}-\partial_{x}^{2}+\varepsilon \partial_{x}^{4}\right)^{-1}: \mathrm{H}^{s} \rightarrow \mathrm{H}^{s+4}
$$

defines a bounded linear operator on the indicated Sobolev spaces. Moreover,

$$
\mathcal{D}(f)=\left(G_{\varepsilon} * f\right)(x)=\int_{\mathbb{R}} G_{\varepsilon}(x-y) f(y) d y, \quad f \in \mathrm{H}^{s},
$$

can be expressed as a convolution with respect to

$$
\begin{aligned}
G_{\varepsilon}(x)= & \frac{1}{2 \sqrt{1-4 \varepsilon}}\left(\frac{\sqrt{1+\sqrt{1-4 \varepsilon}}}{\sqrt{2}} e^{-\sqrt{2 /(1+\sqrt{1-4 \varepsilon})}|x|}\right. \\
& \left.-\frac{\sqrt{2 \varepsilon}}{\sqrt{1+\sqrt{1-4 \varepsilon}}} e^{-\sqrt{(1+\sqrt{1-4 \varepsilon}) / 2 \varepsilon}|x|}\right) .
\end{aligned}
$$

To show the existence of a solution to the regularised problem (3.1), we apply the operator of the above lemma to the regularised problem (3.1). A standard application of the contraction mapping theorem leads to the following result.

THEOREM 3.1. For each initial data $z_{0}=\left(\begin{array}{c}u_{0} \\ \gamma_{0}\end{array}\right) \in H^{s} \times H^{s}, s \geq 1$, there is a $T>0$ depending only on the norm of $z_{0}$ in $H^{s} \times H^{s}$, and a unique solution $z=\left(\begin{array}{l}u \\ \gamma\end{array}\right)$ to (3.1) such that $z(x, t) \in \mathrm{C}\left([0, \mathrm{~T}) ; \mathrm{H}^{s} \times \mathrm{H}^{s}\right)$ satisfies (3.1) in the sense of distributions. If $s \geq 2$, the solution $z(x, t) \in C^{\infty}\left([0, \infty) ; H^{s} \times H^{s}\right)$ exists globally in the time. In particular, when $s \geq 4$, the corresponding solution is a classical global solution.

Next, we consider the norms of solutions by energy estimates.

LEMMA 3.2. Suppose that for some $s \geq 4$, the function $z=\left(\begin{array}{l}u \\ \gamma\end{array}\right)$ is a solution of (3.1) corresponding to the initial data $z_{0}=\left(\begin{array}{l}u_{0} \\ \gamma_{0}\end{array}\right)$ in $H^{s} \times H^{s}$. Then the following hold:

$$
\begin{aligned}
\|u\|_{H^{1}}^{2}+\|\gamma\|_{H^{1}}^{2} & \leq \int_{\mathbb{R}}\left(u^{2}+u_{x}^{2}+\varepsilon u_{x x}^{2}+\gamma^{2}+\gamma_{x}^{2}+\varepsilon \gamma_{x x}^{2}\right) d x \\
& =\int_{\mathbb{R}}\left(u_{0}^{2}+u_{0 x}^{2}+\varepsilon u_{0 x x}^{2}+\gamma_{0}^{2}+\gamma_{0 x}^{2}+\varepsilon \gamma_{0 x x}^{2}\right) d x .
\end{aligned}
$$


For any real number $q \in(0, s-1]$, there exists a constant $c$ depending only on $q$, such that

$$
\begin{aligned}
& \int_{\mathbb{R}}\left(\left(\Lambda^{q+1} u\right)^{2}+\left(\Lambda^{q+1} \gamma\right)^{2}\right) d x \\
& \quad \leq \int_{\mathbb{R}}\left(\left(\Lambda^{q+1} u_{0}\right)^{2}+\varepsilon\left(\Lambda^{q} u_{0 x x}\right)^{2}+\left(\Lambda^{q+1} \gamma_{0}\right)^{2}+\left(\Lambda^{q} \gamma_{0 x x}\right)^{2}\right) d x \\
& \quad+c \int_{0}^{t}\left(\left\|u_{x}\right\|_{L^{\infty}}+\left\|\gamma_{x}\right\|_{L^{\infty}}+\|u\|_{L^{\infty}}+\|\gamma\|_{L^{\infty}}\right)\left(\|\gamma\|_{H^{q+1}}^{2}+\|u\|_{H^{q+1}}^{2}\right) d \tau .
\end{aligned}
$$

For any real number $q \in[0, s-1]$, there exists a constant $c$ such that

$$
\begin{gathered}
(1-2 \varepsilon)\left\|u_{t}\right\|_{H^{q}} \leq c\left(\|u\|_{H^{1}}\|u\|_{H^{q+1}}+\|\gamma\|_{H^{1}}\|\gamma\|_{H^{q+1}}\right), \\
(1-2 \varepsilon)\left\|\gamma_{t}\right\|_{H^{q}} \leq c\left(\|u\|_{H^{1}}\|\gamma\|_{H^{q+1}}\right) .
\end{gathered}
$$

Proof. Multiplying the first equation in (3.1) by $u$ and then integrating by parts, we get

$$
\frac{d}{d t} \int_{\mathbb{R}}\left(u^{2}+u_{x}^{2}+\varepsilon u_{x x}^{2}\right) d x=-\int_{\mathbb{R}} 2 u \gamma \gamma_{x}+2 u \gamma_{x x} \gamma_{x} d x
$$

Multiplying the second equation in (3.1) by $\gamma$ and then integrating by parts, we obtain

$$
\begin{aligned}
& \frac{d}{d t} \int_{\mathbb{R}}\left(\gamma^{2}+\gamma_{x}^{2}+\varepsilon \gamma_{x x}^{2}\right) d x \\
& \quad=-2 \int_{\mathbb{R}}\left(\left(\gamma-\gamma_{x x}\right) u\right)_{x} \gamma d x=\int_{\mathbb{R}} 2 u \gamma \gamma_{x}-2 u \gamma_{x x} \gamma_{x}
\end{aligned}
$$

Combining the above two equalities, we have

$$
\frac{d}{d t} \int_{\mathbb{R}}\left(u^{2}+u_{x}^{2}+\varepsilon u_{x x}^{2}+\gamma^{2}+\gamma_{x}^{2}+\varepsilon \gamma_{x x}^{2}\right) d x=0,
$$

which implies the equality (3.2).

For any $q \in(0, s-1]$, applying $\left(\Lambda^{q} u\right) \Lambda^{q}$ to the first equation in (3.1) and integrating by parts, we get

$$
\begin{aligned}
\frac{1}{2} \frac{d}{d t} \int_{\mathbb{R}}\left[\left(\Lambda^{q} u\right)^{2}+\left(\Lambda^{q} u_{x}\right)^{2}+\varepsilon\left(\Lambda^{q} u_{x x}\right)^{2}\right] d x \\
=-3 \int_{\mathbb{R}} \Lambda^{q} u \Lambda^{q}\left(u u_{x}\right) d x+\int_{\mathbb{R}} \Lambda^{q} u \Lambda^{q}\left(u u_{x x x}\right) d x+\int_{\mathbb{R}} \Lambda^{q} u \Lambda^{q}\left(2 u_{x} u_{x x}\right) d x \\
\quad-\int_{\mathbb{R}} \Lambda^{q} u \Lambda^{q}\left(\gamma \gamma_{x}\right) d x+\int_{\mathbb{R}} \Lambda^{q} u \Lambda^{q}\left(\gamma_{x} \gamma_{x x}\right) d x \\
=-2 \int_{\mathbb{R}} \Lambda^{q} u \Lambda^{q}\left(u u_{x}\right) d x-\int_{\mathbb{R}} \Lambda^{q+1} u \Lambda^{q+1}\left(u u_{x}\right) d x+\frac{1}{2} \int_{\mathbb{R}} \Lambda^{q}\left(u_{x}^{2}\right) \Lambda^{q}\left(u_{x}\right) d x \\
\quad-\frac{1}{2} \int_{\mathbb{R}} \Lambda^{q} u \Lambda^{q}\left(\gamma^{2}\right)_{x} d x+\frac{1}{2} \int_{\mathbb{R}} \Lambda^{q} u \Lambda^{q}\left(\gamma_{x}^{2}\right)_{x} d x .
\end{aligned}
$$


It follows from (2.1)-(2.2) that there exists a constant $c$ such that

$$
\begin{aligned}
& \frac{d}{d t} \int_{\mathbb{R}}\left[\left(\Lambda^{q+1} u\right)^{2}+\varepsilon\left(\Lambda^{q} u_{x x}\right)^{2}\right] d x \\
& \quad \leq c\left(\left\|u_{x}\right\|_{L^{\infty}}+\left\|\gamma_{x}\right\|_{L^{\infty}}+\|u\|_{L^{\infty}}+\|\gamma\|_{L^{\infty}}\right)\left(\|\gamma\|_{H^{q+1}}^{2}+\|u\|_{H^{q+1}}^{2}\right) .
\end{aligned}
$$

Similar to the estimate of $u$, we can obtain

$$
\begin{aligned}
& \frac{d}{d t} \int_{\mathbb{R}}\left[\left(\Lambda^{q+1} \gamma\right)^{2}+\varepsilon\left(\Lambda^{q} \gamma_{x x}\right)^{2}\right] d x \\
& \quad \leq c\left(\left\|u_{x}\right\|_{L^{\infty}}+\left\|\gamma_{x}\right\|_{L^{\infty}}+\|u\|_{L^{\infty}}+\|\gamma\|_{L^{\infty}}\right)\left(\|\gamma\|_{H^{q+1}}^{2}+\|u\|_{H^{q+1}}^{2}\right) .
\end{aligned}
$$

By the above two inequalities, we have

$$
\begin{aligned}
& \frac{d}{d t} \int_{\mathbb{R}}\left[\left(\Lambda^{q+1} u\right)^{2}+\varepsilon\left(\Lambda^{q} \gamma_{x x}\right)^{2}+\left(\Lambda^{q+1} \gamma\right)^{2}+\varepsilon\left(\Lambda^{q} \gamma_{x x}\right)^{2}\right] d x \\
& \quad \leq c\left(\left\|u_{x}\right\|_{L^{\infty}}+\left\|\gamma_{x}\right\|_{L^{\infty}}+\|u\|_{L^{\infty}}+\|\gamma\|_{L^{\infty}}\right)\left(\|\gamma\|_{H^{q+1}}^{2}+\|u\|_{H^{q+1}}^{2}\right) .
\end{aligned}
$$

Integrating with respect to $t$ on both sides of the above inequality leads to the inequality (3.2).

Applying the operator $\Lambda^{-2}$ to both sides of the first equation in (3.1), we get

$$
\begin{aligned}
& (1-\varepsilon) u_{t}-\varepsilon u_{x x t}+u u_{x} \\
& \quad=\left(I-\partial_{x}^{2}\right)^{-1}\left[-\varepsilon u_{t}+\partial_{x}\left(-u^{2}-\frac{u_{x}^{2}}{2}-\frac{\gamma^{2}}{2}+\frac{\gamma_{x}^{2}}{2}\right)\right] .
\end{aligned}
$$

Then applying $\Lambda^{q} u_{t} \Lambda^{q}$ to both sides of (3.6), we obtain

$$
\begin{aligned}
& \int_{\mathbb{R}}\left[(1-\varepsilon)\left(\Lambda^{q} u_{t}\right)^{2}+\varepsilon\left(\Lambda^{q} u_{x t}\right)^{2}+\Lambda^{q} u_{t} \Lambda^{q} u u_{x}\right] d x \\
& \quad=\int_{\mathbb{R}} \Lambda^{q} u_{t}\left(I-\partial_{x}^{2}\right)^{-1} \Lambda^{q}\left[-\varepsilon u_{t}+\partial_{x}\left(-u^{2}-\frac{u_{x}^{2}}{2}-\frac{\gamma^{2}}{2}+\frac{\gamma_{x}^{2}}{2}\right)\right] d x
\end{aligned}
$$

Note that there exists a constant $c$ such that

$$
\left.\left(1+\xi^{2}\right)^{q} \leq c\left[\left(1+(\xi-\eta)^{2}\right)^{q}\right]+\left(1+\eta^{2}\right)^{q}\right]
$$

holds, for any $q>0, \xi, \eta \in \mathbb{R}$. By Schwarz's and Young's inequalities, one can obtain (3.4). Similar to (3.6), we have

$$
(1-\varepsilon) \gamma_{t}-\varepsilon \gamma_{x x t}=\left(I-\partial_{x}^{2}\right)^{-1}\left[-\varepsilon \gamma_{t}-\partial_{x}\left(\left(\gamma-\gamma_{x x}\right) u\right)\right]
$$

Similarly, one also can estimate the norm of $\gamma_{t}$ of (3.4). This completes the proof of the lemma.

We also need the properties of the initial data $u_{\varepsilon 0}$ in the following lemma, which is similar to that of Lemma 5 in [1].

LEMMA 3.3. Let $u_{\varepsilon 0}$ be the convolution $u_{\varepsilon 0}=\phi_{\varepsilon} * u_{0}$ of the functions $\phi_{\varepsilon}(x)=$ $\varepsilon^{-1 / 4} \phi\left(\varepsilon^{-1 / 4} x\right)$ and $u_{0}$ such that the Fourier transform $\hat{\phi}$ of $\phi$ satisfies $\hat{\phi} \in \mathrm{C}_{c}^{\infty}, \hat{\phi}(\xi) \geq 0$ 
and $\hat{\phi}(\xi)=1$ for any $\xi \in(-1,1)$. Then the following estimates hold for any $\varepsilon$ with $0<\varepsilon<\frac{1}{4}$.

$$
\begin{aligned}
\left\|u_{\varepsilon 0}\right\|_{H^{q}} \leq c, & \text { if } q \leq s, \\
\left\|u_{\varepsilon 0}\right\|_{H^{q}} \leq c \varepsilon^{\frac{s-q}{4}}, & \text { if } q>s .
\end{aligned}
$$

Similarly, we can also define $\gamma_{\varepsilon 0}$ and get the estimates of $\gamma_{\varepsilon 0}$. For this, we just replace $u_{\varepsilon} 0$ by $\gamma_{\varepsilon 0}$ and obtain the same estimates as that of $u_{\varepsilon 0}$.

4. The existence of low regularity weak solutions. From the above preparations, the following property holds for the weak solutions:

THEOREM 4.1. Suppose that $u_{0}(x)$ and $\gamma_{0}(x)$ are functions of Sobolev space $\mathrm{H}^{s}$ for $s \in[1,3 / 2]$ such that $\left\|u_{0 x}\right\|_{\mathrm{L}^{\infty}}<\infty$ and $\left\|\gamma_{0 x}\right\|_{\mathrm{L}^{\infty}}<\infty$. Let $u_{\varepsilon 0}$ and $\gamma_{\varepsilon 0}$ be defined as in Lemma 3.3. Then there exist constants $T>0$ and $c>0$ being independent of $\varepsilon$ such that the corresponding solution $z_{\varepsilon}$ of (3.1) satisfies $\left\|u_{\varepsilon x}\right\|_{\mathrm{L}^{\infty}}+\left\|\gamma_{\varepsilon x}\right\|_{\mathrm{L}^{\infty}} \leq$ c for any $t \in[0, T)$.

Proof. Let us start from the first equation of the regularised problem (3.1) with $u=u_{\varepsilon}$. Differentiating with respect to $x$ on both sides of (3.6), one obtain

$$
\begin{aligned}
& (1-\varepsilon) u_{t x}-\varepsilon u_{x x x t}+u u_{x x}+\frac{u_{x}^{2}}{2} \\
& \quad=\left(I-\partial_{x}^{2}\right)^{-1}\left(-\varepsilon u_{t x}-u^{2}-\frac{u_{x}^{2}}{2}-\frac{\gamma^{2}}{2}+\frac{\gamma_{x}^{2}}{2}\right)+\left(u^{2}+\frac{\gamma^{2}}{2}-\frac{\gamma_{x}^{2}}{2}\right) .
\end{aligned}
$$

Let $n>0$ be an integer. Then multiplying the above equation by $\left(u_{x}\right)^{2 n+1}$ to integrate with respect to $x$ yields the equality

$$
\begin{aligned}
& \frac{1-\varepsilon}{2 n+2} \frac{d}{d t} \int_{\mathbb{R}}\left(u_{x}\right)^{2 n+2} d x-\varepsilon \int_{\mathbb{R}}\left(u_{x}\right)^{2 n+1} u_{x x x t} d x+\frac{n}{2 n+2} \int_{\mathbb{R}}\left(u_{x}\right)^{2 n+3} d x \\
& =\int_{\mathbb{R}}\left(u_{x}\right)^{2 n+1} \Lambda^{-2}\left(-\varepsilon u_{t x}-u^{2}-\frac{u_{x}^{2}}{2}-\frac{\gamma^{2}}{2}+\frac{\gamma_{x}^{2}}{2}\right) d x \\
& \quad+\int_{\mathbb{R}}\left(u_{x}\right)^{2 n+1}\left(\left(u^{2}+\frac{\gamma^{2}}{2}-\frac{\gamma_{x}^{2}}{2}\right) d x .\right.
\end{aligned}
$$

Set $g=\Lambda^{-2}\left(-\varepsilon u_{t x}-u^{2}-\frac{u_{x}^{2}}{2}-\frac{\gamma^{2}}{2}+\frac{\gamma_{x}^{2}}{2}\right)$. By Hölder's inequality, we get

$$
\begin{aligned}
& \frac{1-\varepsilon}{2 n+2} \frac{d}{d t} \int_{\mathbb{R}}\left(u_{x}\right)^{2 n+2} d x \\
& \left.\leq\left(\int_{\mathbb{R}}\left|u_{x}\right|^{2 n+2} d x\right)^{\frac{2 n+1}{2 n+2}}\right)\left[\varepsilon\left(\int_{\mathbb{R}}\left|u_{x x x t}\right|^{2 n+2} d x\right)^{\frac{1}{2 n+2}}+\left(\int_{\mathbb{R}}|u|^{4 n+4} d x\right)^{\frac{1}{2 n+2}}\right. \\
& \left.\quad+\frac{1}{2}\left(\int_{\mathbb{R}}|\gamma|^{4 n+4} d x\right)^{\frac{1}{2 n+2}}+\frac{1}{2}\left(\int_{\mathbb{R}}\left|\gamma_{x}\right|^{4 n+4} d x\right)^{\frac{1}{2 n+2}}\right] \\
& \quad+\left(\int_{\mathbb{R}}|g|^{2 n+2} d x\right)^{\frac{1}{2 n+2}}+\frac{n}{2 n+2}\left\|u_{x}\right\|_{L^{\infty}} \int_{\mathbb{R}}\left|u_{x}\right|^{2 n+2} d x,
\end{aligned}
$$


or

$$
\begin{aligned}
(1-\varepsilon) & \frac{d}{d t}\left(\int_{\mathbb{R}}\left|u_{x}\right|^{2 n+2} d x\right)^{\frac{1}{2 n+2}} \\
\leq & \varepsilon\left(\int_{\mathbb{R}}\left|u_{x x x t}\right|^{2 n+2} d x\right)^{\frac{1}{2 n+2}}+\left(\int_{\mathbb{R}}|u|^{4 n+4} d x\right)^{\frac{1}{2 n+2}} \\
+ & \frac{1}{2}\left(\int_{\mathbb{R}}|\gamma|^{4 n+4} d x\right)^{\frac{1}{2 n+2}}+\frac{1}{2}\left(\int_{\mathbb{R}}\left|\gamma_{x}\right|^{4 n+4} d x\right)^{\frac{1}{2 n+2}} \\
+ & \left(\int_{\mathbb{R}}|g|^{2 n+2} d x\right)^{\frac{1}{2 n+2}}+\frac{n\left\|u_{x}\right\|_{L^{\infty}}}{2 n+2}\left(\int_{\mathbb{R}}\left|u_{x}\right|^{2 n+2} d x\right)^{\frac{1}{2 n+2}} .
\end{aligned}
$$

Since $\|f\|_{L^{p}} \rightarrow\|f\|_{L^{\infty}}$ as $p \rightarrow \infty$ for any $f \in L^{\infty} \bigcap L^{2}$, integrating with respect to $t$ and taking the limit as $p \rightarrow \infty$ on both sides of the above inequality leads to the estimate

$$
\begin{aligned}
& (1-\varepsilon)\left\|u_{x}\right\|_{L^{\infty}} \\
& \quad \leq(1-\varepsilon)\left\|u_{0 x}\right\|_{L^{\infty}}+\int_{0}^{t}\left[\varepsilon\left\|u_{x x x t}\right\|_{L^{\infty}}+c\left(\left\|u^{2}\right\|_{L^{\infty}}+\|g\|_{L^{\infty}}\right.\right. \\
& \left.\left.\quad+\left\|\gamma^{2}\right\|_{L^{\infty}}\right)+\frac{1}{2}\left\|\gamma_{x}^{2}\right\|_{L^{\infty}}+\frac{1}{2}\left\|u_{x}\right\|_{L^{\infty}}^{2}\right] d \tau .
\end{aligned}
$$

Note that if $p(x):=\frac{1}{2} e^{-|x|}, x \in \mathbb{R}$, then $\Lambda^{-2} f=p * f$ for all $f \in L^{2}$. Thus,

$$
\|g\|_{L^{\infty}} \leq \tilde{c}\left(\left\|u_{t}\right\|_{L^{2}}+\|u\|_{L^{2}}^{2}+\left\|u_{x}\right\|_{L^{2}}^{2}+\|\gamma\|_{L^{2}}^{2}+\left\|\gamma_{x}\right\|_{L^{2}}^{2}\right),
$$

where the constant $\tilde{c}$ is independent of $\varepsilon$. Therefore, it follows from (3.2), (3.4) and (3.8) that

$$
\|g\|_{L^{\infty}} \leq c_{1}\left(\left\|u_{\varepsilon 0}\right\|_{H^{1}}+\left\|\gamma_{\varepsilon} 0\right\|_{H^{1}}\right)^{2} \leq c_{2}
$$

where constants $c_{1}, c_{2}$ are independent of $\varepsilon$. Moreover, for any fixed $r \in\left(\frac{1}{2}, 1\right)$, there is a constant $c_{r}$ such that $\left\|u_{x x x t}\right\|_{L^{\infty}} \leq c_{r}\left\|u_{x x x t}\right\|_{H^{r}} \leq c_{r}\left\|u_{t}\right\|_{H^{r+3}}$. This combining (3.2) with (3.4) yields

$$
\left\|u_{x x x t}\right\|_{L^{\infty}} \leq c\|u\|_{H^{r+4}} .
$$

Similarly, we have

$$
(1-\varepsilon) \gamma_{t x}-\varepsilon \gamma_{x x x t}=\left(I-\partial_{x}^{2}\right)^{-1}\left[-\varepsilon \gamma_{x t}-\left(\gamma-\gamma_{x x}\right) u\right]+\left(\gamma-\gamma_{x x}\right) u .
$$

By integration by parts and Hölder's inequality, we get

$$
\begin{aligned}
(1-\varepsilon) & \frac{d}{d t}\left(\int_{\mathbb{R}}\left|\gamma_{x}\right|^{2 n+2} d x\right)^{\frac{1}{2 n+2}} \\
\leq & \varepsilon\left(\int_{\mathbb{R}}\left|\gamma_{x x x t}\right|^{2 n+2} d x\right)^{\frac{1}{2 n+2}}+\left(\int_{\mathbb{R}}|u|^{4 n+4} d x\right)^{\frac{1}{4 n+4}}\left(\int_{\mathbb{R}}|\gamma|^{4 n+4} d x\right)^{\frac{1}{4 n+4}} \\
& +\left(\int_{\mathbb{R}}\left|u_{x} \gamma_{x}\right|^{2 n+2} d x\right)^{\frac{1}{2 n+2}}+\left(\int_{\mathbb{R}}|h|^{2 n+2} d x\right)^{\frac{1}{2 n+2}}
\end{aligned}
$$


where $h=\Lambda^{-2}\left(-\varepsilon \gamma_{t x}-u \gamma-u_{x} \gamma_{x}\right)$. Thus,

$$
\begin{aligned}
& (1-\varepsilon)\left\|\gamma_{x}\right\|_{L^{\infty}} \\
& \leq(1-\varepsilon)\left\|\gamma_{0 x}\right\|_{L^{\infty}}+\int_{0}^{t}\left[\varepsilon\left\|\gamma_{x x x t}\right\|_{L^{\infty}}\right. \\
& \left.\quad+c\left(\|u\|_{L^{\infty}}\|\gamma\|_{L^{\infty}}+\|g\|_{L^{\infty}}\right)+\left\|\gamma_{x}\right\|_{L^{\infty}}\left\|u_{x}\right\|_{L^{\infty}}\right] d \tau .
\end{aligned}
$$

Like the situation with the function $g$, we get $\|h\|_{L^{\infty}} \leq c_{2}$ and

$$
\left\|\gamma_{x x x t}\right\|_{L^{\infty}} \leq c\|\gamma\|_{H^{r+4}}
$$

Applying Gronwall's inequality to (3.3) with $q=r+3$ and $u=u_{\varepsilon}$, we obtain

$$
\begin{aligned}
& \|u\|_{H^{r+4}}^{2}+\|\gamma\|_{H^{r+4}}^{2} \\
& \quad \leq\left(\int_{\mathbb{R}}\left(\left(\Lambda^{r+4} u_{0}\right)^{2}+\varepsilon\left(\Lambda^{r+3} u_{0 x x}\right)^{2}+\left(\Lambda^{r+4} \gamma_{0}\right)^{2}+\left(\Lambda^{r+3} \gamma_{0 x x}\right)^{2}\right) d x\right) \\
& \quad \exp \left(c \int_{0}^{t}\left(\left\|u_{x}\right\|_{L^{\infty}}+\left\|\gamma_{x}\right\|_{L^{\infty}}+c\right) d \tau\right) .
\end{aligned}
$$

Then it follows from (3.9), (4.2) and (4.4) that

$$
\begin{aligned}
& \left\|u_{x x x t}\right\|_{L^{\infty}}+\left\|\gamma_{x x x t}\right\|_{L^{\infty}} \\
& \quad \leq c \varepsilon^{\frac{s-r-4}{4}} \exp \left(c \int_{0}^{t}\left(\left\|u_{x}\right\|_{L^{\infty}}+\left\|\gamma_{x}\right\|_{L^{\infty}}+c_{3}\right) d \tau\right) .
\end{aligned}
$$

Since $0<\varepsilon<\frac{1}{4}$, combining (3.2), (4.1), (4.3) and (4.5) to obtain

$$
\begin{aligned}
& \left\|u_{x}\right\|_{L^{\infty}}+\left\|\gamma_{x}\right\|_{L^{\infty}} \\
& \leq\left(\left\|u_{0 x}\right\|_{L^{\infty}}+\left\|\gamma_{0 x}\right\|_{L^{\infty}}\right)+\frac{4 c}{3} \int_{0}^{t} \leq\left[\varepsilon^{\frac{s-r}{4}} \exp \left(c \int_{0}^{\tau}\left(\left\|u_{x}\right\|_{L^{\infty}}+\left\|\gamma_{x}\right\|_{L^{\infty}}\right) d s\right)\right. \\
& \left.\quad+\frac{1}{2}\left(\left\|u_{x}\right\|_{L^{\infty}}+\left\|\gamma_{x}\right\|_{L^{\infty}}\right)^{2}+1\right] d \tau .
\end{aligned}
$$

Applying the contraction mapping principle yields that there exists a $T>0$ such that the integral equation

$$
\psi(t)=\left(\left\|u_{0 x}\right\|_{L^{\infty}}+\left\|\gamma_{0 x}\right\|_{L^{\infty}}\right)+\frac{4 c}{3} \int_{0}^{t}\left[\exp \left(c \int_{0}^{\tau} \psi(s) d s\right)+\frac{1}{2} \psi^{2}(\tau)+1\right] d \tau
$$

has a unique solution $\psi(t) \in C[0, T]$. The comparison principle leads to the estimate $\left\|u_{x}\right\|_{L^{\infty}}+\left\|\gamma_{x}\right\|_{L^{\infty}} \leq \psi(t)$ for any $t \in[0, T]$, which implies the conclusion of the theorem. This completes the proof of the theorem.

As a direct result of Theorem 4.1, one can estimate norms of $u=u_{\varepsilon}$ and $\gamma=\gamma_{\varepsilon}$. Using (3.3), (3.4), (3.5), (3.8) and (3.9) and Gronwall's inequality, we deduce that there is a constant $c>0$ such that the inequalities

$$
\begin{aligned}
& \left\|u_{\varepsilon}\right\|_{H^{q}}+\left\|\gamma_{\varepsilon}\right\|_{H^{q}}=\|u\|_{H^{q}}+\|\gamma\|_{H^{q}} \\
& \quad \leq c \exp c \int_{0}^{t}\left(\left\|u_{x}\right\|_{L^{\infty}}+\left\|\gamma_{x}\right\|_{L^{\infty}}\right) d \tau \leq c \exp c \int_{0}^{t} f(\tau) d \tau
\end{aligned}
$$


and

$$
\left\|u_{\varepsilon t}\right\|_{H^{r}}+\left\|\gamma_{\varepsilon t}\right\|_{H^{r}}=\left\|u_{t}\right\|_{H^{r}}+\left\|\gamma_{t}\right\|_{H^{r}} \leq c \exp c \int_{0}^{t} f(\tau) d \tau,
$$

hold for any $q \in(0, s], r \in(0, s-1]$ and any $t \in[0, T]$. Then it follows from EberleinShmulyan theorem, that there are subsequences of $\left\{u_{\varepsilon}\right\}$ and $\left\{\gamma_{\varepsilon}\right\}$, denoted by $\left\{u_{\varepsilon_{n}}\right\}$ and $\left\{\gamma_{\varepsilon_{n}}\right\}$ respectively, such that $\left\{u_{\varepsilon_{n}}\right\}$ and $\left\{\gamma_{\varepsilon_{n}}\right\}$ are weakly convergent to the function $u(x, t)$ and $\gamma(x, t)$ in $L^{2}\left([0, T], H^{s}\right)$, respectively. Their temporal derivatives $\left\{u_{\varepsilon_{n}}\right\}$ and $\left\{\gamma_{\varepsilon_{n}}\right\}$ weakly converge to the temporal derivatives $u_{t}$ and $\gamma_{t}$ in $L^{2}\left([0, T], H^{s-1}\right)$, respectively. Moreover, for any real number $R>0$, it follows from Lions-Aubin's compactness theorem [27], $\left\{u_{\varepsilon_{n}}\right\}$ and $\left\{\gamma_{\varepsilon_{n}}\right\}$ are convergent to the function $u$ and $\gamma$ strongly in the space $L^{2}\left([0, T], H^{q}(-R, R)\right)$ for any $q \in[0, s)$, respectively. And $\left\{u_{\varepsilon_{n}}\right\}$ and $\left\{\gamma_{\varepsilon_{n}}\right\}$ converge to $u_{t}$ and $\gamma_{t}$ strongly in the space $L^{2}\left([0, T], H^{r}(-R, R)\right)$ for any $r \in[0, s-1)$, respectively.

Therefore, one can obtain the existence of a weak solution to the Cauchy problem (2.3) as follows:

THEOREM 4.2. Suppose that $z_{0}=\left(\begin{array}{l}u_{0} \\ \gamma_{0}\end{array}\right)$ is a pair of functions of Sobolev space $H^{s} \times H^{s}$ for $s \in(1,3 / 2]$ satisfying $\left\|u_{0 x}\right\|_{L^{\infty}}<\infty$ and $\left\|\gamma_{0 x}\right\|_{L^{\infty}}<\infty$. Then there is a $T>0$ such that the Cauchy problem (2.3) with the initial data $z_{0}=\left(\begin{array}{l}u_{0} \\ \gamma_{0}\end{array}\right)$ has a solution $z=\left(\begin{array}{l}u \\ \gamma\end{array}\right)$ such that $z(x, t) \in L^{2}\left([0, T] ; H^{s} \times H^{s}\right)$ in the sense of distributions, and $u_{x} \in L^{\infty}([0, T] \times$ $\mathbb{R}), \gamma_{x} \in L^{\infty}([0, T] \times \mathbb{R})$.

Proof. It follows from Theorem 4.1 that $\left\{u_{\varepsilon_{n} x}\right\}$ and $\left\{\gamma_{\varepsilon_{n} x}\right\}$ are bounded in the space $L^{\infty}$, respectively. Hence, the sequences $\left\{u_{\varepsilon_{n}}^{2}\right\},\left\{u_{\varepsilon_{n} x}^{2}\right\},\left\{\gamma_{\varepsilon_{n}}^{2}\right\},\left\{\gamma_{\varepsilon_{n} x}^{2}\right\},\left\{u_{\varepsilon_{n}} \gamma_{\varepsilon_{n}}\right\}$ and $\left\{u_{\varepsilon_{n} x} \gamma_{\varepsilon_{n} x}\right\}$ weakly converge to $u^{2}, u_{x}^{2}, \gamma^{2}, \gamma_{x}^{2}, u \gamma$ and $u_{x} \gamma_{x}$ in $L^{2}\left([0, T], H^{r}(-R, R)\right)$ for any $r \in[0, s-1)$, respectively. Therefore, $u$ and $\gamma$ satisfy the equation as follows:

$$
\begin{aligned}
\int_{0}^{T} & \int_{\mathbb{R}} u\left(\varphi_{t}-\varphi_{x x t}\right) d x d t \\
\quad= & \int_{0}^{T} \int_{\mathbb{R}}\left[\left(-\frac{3}{2} u^{2}-\frac{1}{2} u_{x}^{2}-\frac{1}{2} \gamma^{2}+\frac{1}{2} \gamma_{x}^{2}\right) \varphi_{x}+\frac{1}{2} u^{2} \varphi_{x x x}\right] d x d t
\end{aligned}
$$

and

$$
\int_{0}^{T} \int_{\mathbb{R}} \gamma\left(\varphi_{t}-\varphi_{x x t}\right) d x d t=\int_{0}^{T} \int_{\mathbb{R}}\left(-\gamma u-\gamma_{x} u_{x}\right) \varphi_{x} d x d t
$$

for any $\varphi \in C_{c}^{\infty}$.

Moreover, $\left\{u_{\varepsilon_{n} x}\right\}$ is a bounded sequence in the space $X^{*}=L^{\infty}([0, T] \times \mathbb{R})$ of $X=$ $L^{1}([0, T] \times \mathbb{R})$, so there is a subsequence of $\left\{u_{\varepsilon_{n} x}\right\}$, still denoted by $\left\{u_{\varepsilon_{n} x}\right\}$, weakly $*$ converging to a function $v$ in $L^{\infty}([0, T] \times \mathbb{R})$. Since $\left\{u_{\varepsilon_{n} x}\right\}$ is also weakly convergent to $u_{x}$ in $L^{2}([0, T] \times \mathbb{R})$, it follows that $u_{x}=v$ almost everywhere. Hence, $u_{x} \in L^{\infty}([0, T] \times \mathbb{R})$. Similarly, we can also obtain $\gamma_{x} \in L^{\infty}([0, T] \times \mathbb{R})$. This completes the proof of the theorem.

ACKNOWLEDGEMENTS. This work was partially supported by NNSFC (No. 10971235), RFDP (No. 200805580014), NCET-08-0579 and the key project of Sun Yat-Sen University. 


\section{REFERENCES}

1. J. L. Bona and R. Smith, The initial value problem for the Korteweg-de Vries equation, Phil. Trans. R. Soc. Lond. A 278 (1975), 555-601.

2. R. Camassa and D. Holm, An integrable shallow water equation with peaked solitons, Phys. Rev. Lett. 71 (1993), 1661-1664.

3. R. Camassa, D. Holm and J. Hyman, A new integrable shallow water equation, $A d v$. Appl. Mech. 31 (1994), 1-33.

4. M. Chen, S-Q. Liu and Y. Zhang, A 2-component generalization of the Camassa-Holm equation and its solutions, Lett. Math. Phys. 75 (2006), 1-15.

5. A. Constantin, The Hamiltonian structure of the Camassa-Holm equation, Expo. Math. 15 (1997), 53-85.

6. A. Constantin, On the scattering problem for the Camassa-Holm equation, Proc. $R$. Soc. Lond. A 457 (2001), 953-970.

7. A. Constantin and D. Lannes, The hydrodynamical relevance of the CamassaHolm and Degasperis-Procesi equations, Arch. Ration. Mech. Anal. 1992 (2009), 165186.

8. A. Constantin and J. Escher, Global existence and blow-up for a shallow water equation, Annali Sci. Norm. Sup. Pisa 26 (1998), 303-328.

9. A. Constantin and J. Escher, Wave breaking for nonlinear nonlocal shallow water equations, Acta Math. 181 (1998), 229-243.

10. A. Constantin and J. Escher, Particle trajectories in solitary water waves, Bull. Amer. Math. Soc. 44 (2007), 423-431.

11. A. Constantin and R. Ivanov, On an integrable two-component Camass-Holm shallow water system, Phys. Lett. A 372 (2008), 7129-7132.

12. A. Constantin and W. Strauss, Stability of peakons, Commun. Pure Appl. Math. 53 (2000), 603-610.

13. H. Dai, Model equations for nonlinear dispersive waves in compressible Mooney-Rivlin rod, Acta Mech. 127 (1998), 193-207.

14. R. Danchin, A few remarks on the Camassa-Holm equation, Differ. Int. Equ. 14 (2001), 953-988.

15. H. R. Dullin, G. A. Gottwald and D. D. Holm, An integral shallow water equation with linear and nonlinear dispersion, Phys. Rev. Lett. 87 (2001), 4501-4504.

16. J. Escher, O. Lechtenfeld and Z. Yin, Well-posedness and blow-up phenomena for the 2-component Camassa-Holm equation, Disc. Cont. Dyn. Syst. 19 (2007), 493513.

17. J. Escher and Z. Yin, Initial boundary value problems of the Camassa-Holm equation, Commun. Part. Differ. Equ. 33 (2008), 377-395.

18. J. Escher and Z. Yin, Initial boundary value problems for nonlinear dispersive wave equations, J. Funct. Anal. 256 (2008), 479-508.

19. G. Falqui, On a Camassa-Holm type equation with two dependent variables, J. Phys. A 39 (2006), 327-342.

20. C. Guan and Z. Yin, Global existence and blow-up phenomena for an integrable two component Camassa-Holm shallow water system, J. Differ. Equ. 248 (2010), 20032014.

21. C. Guan, K. H. Karlsen and Z. Yin, Well-posedness and blow-up phenomena for a modified two-component Camassa-Holm equation, in Proceedings of the 2008-2009 special year in nonlinear partial differential equations, vol. 526 (Holden H. and Karlsen K. H., Editors) (Contemporary Mathematics, American Mathematical Society, Providence, RI, 2010), 199-220.

22. D. Holm and R. Ivanov, Two-component $\mathrm{CH}$ system: Inverse scattering, peakons and geometry, arXiv:1009.5374v1 [nlin.SI] (27 Sep. 2010).

23. D. Holm, L. Naraigh and C. Tronci, Singular solution of a modified two-component Camassa-Holm equation, Phys. Rev. E 79 (2009), 1-13.

24. R. Ivanov, Water waves and integrability, Philos. Trans. R. Soc. Lond. A 365 (2007), $2267-2280$.

25. T. Kato and G. Ponce, Commutator estimates and Navier-Stokes equations, Commun. Pure Appl. Math. 41 (1988), 203-208. 
26. Y. Li and P. Oliver, Well-posedness and blow-up solutions for an integrable nonlinear dispersive model wave equation, J. Differ. Equ. 162 (2000), 27-63.

27. J.-L. Lions, Quelques Méthodes de Résolution des Problèmes aux Limites Nonlinéaires (Dunod, Gauthier-Villars, Paris, 1969).

28. Z. Yin, Well-posedness, global existence and blowup phenomena for an integrable shallow water equation, Disc. Cont. Dyn. Syst. 10 (2004), 393-411. 